\title{
ANTECEDENTS AND CONSEQUENCES OF CONSUMER PERCEPTION OF PRODUCT INNOVATIVENESS
}

\author{
Ben Lowe, University of Kent, United Kingdom \\ Frank Alpert, University of Queensland, Australia
}

\begin{abstract}
Scholarship in innovation has increasingly sought to understand diffusion of new products by examining individual consumer behaviour processes (Alexander, Lynch, and Wang, 2008; Hoeffler 2003; Moreau, Lehman, and Markman, 2001), whereby an innovation is only new if it is perceived to be new by consumers (Rogers 2003). But how new is "new"? Or, in terms of this study's focus, how innovative is an innovation? Currently, the literature contains neither complete agreement as to how to define and measure perceived innovativeness nor an existing model of its antecedents and consequences. This study addresses two main research questions: (1) What is perceived innovativeness and how should researchers define, conceptualize and measure it? and (2) What are the antecedents and consequences of perceived innovativeness, defined here as Consumer Perception of Product Innovativeness (CPPI), and how can the relevant constructs be put together into a logical and useful theory? This study contributes to the literature on innovation management by addressing calls from significant and highly cited studies in the field to "...examine the dimensions and effects of the newness of products to their prospective customers." (Danneels and Kleinschmidt, 2001, p.371).
\end{abstract}

To gain a deeper understanding of the consumer's perspective and to specify the domain of the construct, the first step of this research comprised qualitative interviews with 20 consumers. Respondents were interviewed to ascertain what they understood innovativeness to be and what makes a product innovative to consumers. Specifically, the qualitative interviews suggest that innovativeness is more than just newness, contrary to some prior research. Thus, a true innovation not only has to be new but also must offer a significant improvement to consumers, and must extend beyond a purely cognitive set of dimensions. It was also found that consumers seemed to rate technology newness as an important influencer of CPPI. To follow up the qualitative study a pilot study was then conducted based on a questionnaire administered to 104 students, quantitatively examining the constructs highlighted by the qualitative research. A big question that remains unanswered is whether and which of these dimensions and related constructs are part of the definition of an innovation, or are better conceived of as proximal antecedents and proximal consequences. That is, the concept of an innovation can be conceived of as a multidimensional construct, or a core unidimensional construct surrounded in its nomological net by proximal antecedents and proximal consequences. Using structural equation modeling the evidence suggests CPPI is a distinct unidimensional construct, explained largely by the three proximal antecedents of perceived concept newness, perceived relative advantage, and perceived technological newness, contrary to some studies in the literature. The initial model was then extended to provide a more complete understanding of the key antecedents and key consequences of CPPI and key moderating factors. It was also tested on 826 consumers using a national panel from a commercial market research organization. To extend the analysis, consumer evaluations of innovations were modeled by decomposing attitudes into their hedonic and utilitarian components, and to complete the hierarchy of effects, the effect of attitudes upon purchase intention. Therefore, this study builds on prior literature by measuring consumers' affective response to innovative products using an established measurement framework (HED-UT), and by viewing these affect variables as a consequence of perceived innovativeness, rather than a dimension of perceived innovativeness, as has been the dominant view in prior, yet untested research. Key moderators are also tested using structural equation modeling.

The findings from this research suggest that CPPI is a unidimensional abstract concept, not a multidimensional concept as much past research implies. This study also shows that consumers evaluate innovations along affective dimensions rather than solely along rational dimensions such as how much time and money it saves, and that affect is a consequence of CPPI rather than a dimension of CPPI. Therefore, the model in this study also implies managers can try to influence CPPI through their marketing communications by attempting to raise CPPI's antecedents. Overall, the theory allows for a new operational definition for CPPI, defined as consumers' overall innovativeness assessment, resulting from perceptions of product concept newness, technology newness, and relative advantage, and influencing consumer utilitarian (cognitive) and hedonic (emotional) response. A key finding of this research relates to the dimensionality of CPPI. The literature contains fragmented definitions of the construct. However, one theme is that innovativeness equates with newness (Hoeffler, 2003). The findings from our study show that perceived "concept newness" is indeed a key proximal antecedent of CPPI, although newness is not a sufficient condition. Other constructs, including perceived relative advantage and perceived technology newness, are also important, and these three constructs together offer a richer explanation of variance in CPPI. In sum, this article presents a theory of consumer perception of product innovativeness, starting with introducing the term consumer perceived product innovativeness. References available upon request. 